\title{
Can high-energy pulses replace methyl bromide?
}

Methyl bromide, the fumigant used in the production and postharvest treatment of more than 60 California crops, is both toxic and volatile. These characteristics - invaluable to growers - have raised environmental and health concerns, making it the target of proposed restrictions and production bans. (Some studies have shown it may damage the earth's protective ozone layer. Others indicate it may cause cancer and birth defects.)

In 1990, the federal Clean Air Act called for a $100 \%$ methyl bromide ban by 2001 to protect the ozone layer. Before that, in 1984, California's Birth Defect Prevention Act mandated studies of the potential health effects of this and other chemicals by 1991. The deadline for methyl bromide studies was later extended to March 1996. Because one study remains unfinished, the fumigant faced suspension this year. However, recent legislation has extended the deadline to December 1997, averting the suspension of its use, at least for now.

UC scientists are investigating a wide spectrum of methyl bromide alternatives ranging from conventional chemicals to organic production methods (see page 24, and California Agriculture, May-June 1994).

Some novel alternatives include the use of electromagnetic energy. UC Davis chemist Manuel Lagunas-Solar, plant pathologist James MacDonald and entomologist Jeffrey Granett are developing three forms of electromagnetic energy for use in different scenarios - radio frequencies to clean up greenhouse soils, microwaves to destroy soilborne pests in large-scale field operations, and ultraviolet (UV) light for postharvest pest control.
The scientists have already achieved some success with two of the techniques: the use of radio frequencies in greenhouse soils and the use of UV light in harvested fruit. They have just begun to test the third technique, using microwaves as a preplant soil treatment. All methods would offer nonpolluting methods to kill pests.

The three techniques use electromagnetic energy differently. The radio frequency method uses an electrical current to heat and pasteurize soil. Relatively expensive, it is appropriate for high-value, low-volume soil applications. UV light targets pest organisms on the surface of fruit, killing them by disrupting their nucleic acids, and therefore cell metabolism and growth. The microwave technique, still under development, will aim energy at cell constituents specific to pest organisms. By creating vibration in these constituents (e.g., carbohydrates, proteins, or lipids) the microwaves will generate enough heat to kill pests without heating the bulk of the soil.

In both the microwave and the UV light methods, the effect is enhanced through the use of energy pulses, peak power compressed into very short bursts. The patented UV process has been tested on table grapes, tomatoes, citrus, stone fruit, spices and water. Patents are pending for the other methods.

How the cost of these methods will compare to methyl bromide fumigation is unknown. "Until we define the specifics of each application we will not be able to evaluate economic competitiveness" says Granett. "The goals are to develop methods that are cheaper and better, and we believe we can reach those goals." - Editor

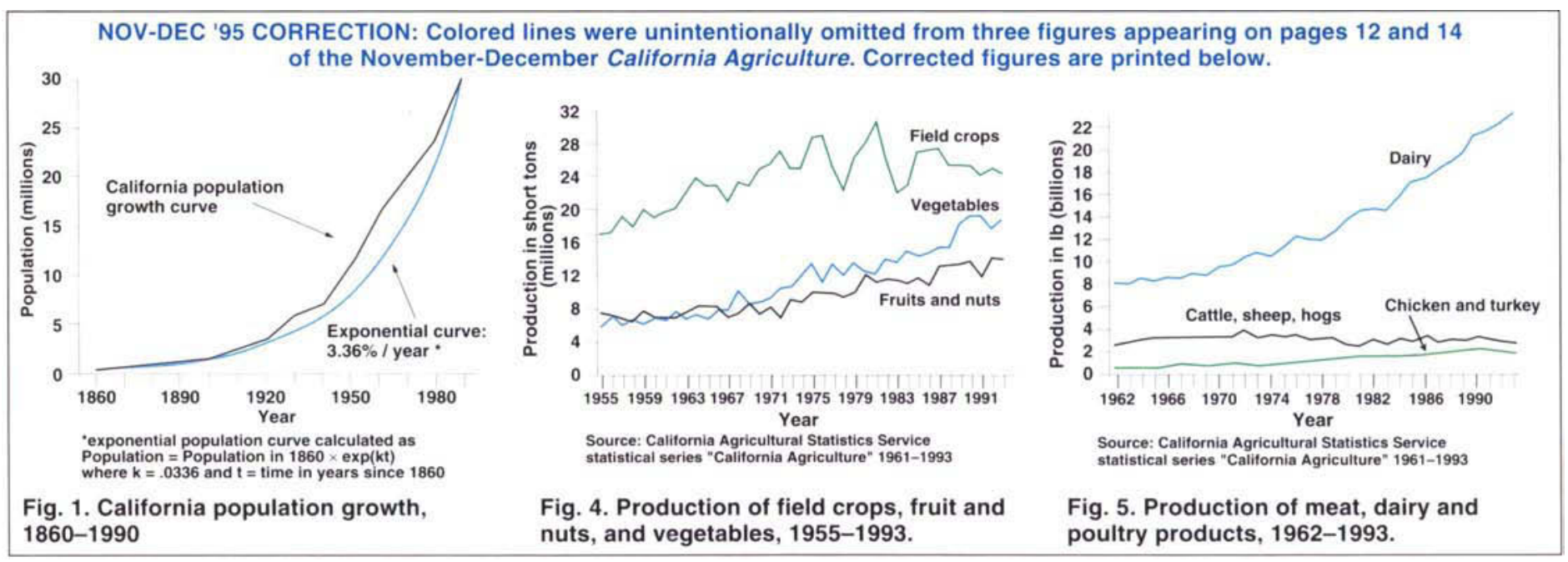

Fig. 1. California population growth, nuts, and vegetables, 1955-1993. 